\title{
UN ÍNDICE PONDERADO DE PRECIOS INDUSTRIALES, 1874-1913*
}

ENRIC MORELLÀ

Universitat Jaume I

La bistoriografia española contemporánea (...) ha revolucionado nuestros conocimientos sobre la evolución de la producción industrial (...) No se puede afirmar nada parecido en relación a los precios (...) Abi dominan las tinieblas ${ }^{1}$.

Cualquier referencia al comportamiento de los precios en la economía española, anterior a la Primera Guerra Mundial, pasa ineludiblemente por el índice de Sardá (1948) para 1812-1890, y desde entonces el que se recoge en el Dictamen de la Comisión del patrón oro. El índice de precios al por mayor de Bustelo y Tortella (1976) para este período es el resultado del enlace entre los dos anteriores. Por otra parte, algunos investigadores han preparado índices de precios basados en estadísticas oficiales de comercio. Así, por ejemplo, Leandro Prados elaboró un índice de precios para las exportaciones y otro para las importaciones a partir de las estadísticas del comercio exterior español y británico ${ }^{2}$. Las estadísticas del comercio de cabotaje también han sido utilizadas para obtener información sobre la evolución de los precios correspondientes ${ }^{3}$. Ninguno de ellos, sin embargo, informa satisfactoriamente sobre el comportamiento de los precios en el sector industrial de la economía española.

* La primera versión de este trabajo (E. Morellà, Precios industriales, 1874-1913, mimeo, Seminario de Historia Económica, Universidad de Valencia) se discutió en el seno del equipo formado con C. Betrán, A. Cubel y D. Tirado, bajo la dirección de Jordi Palafox. El texto se ha enriquecido también con las críticas y valiosa información de Sebastián Coll. Agradezco a todos sus comentarios y sugerencias, que en ningún caso excusan mi responsabilidad.

1 Carreras (1989), p. 183.

2 Prados (1982), pp. 95-98.

3 Arenales (1976), pp. 1111-1122.

Revista de Historia Económica

Año XV, Otoño-Invierno 1997, N."3. 
Los dos primeros comparten un serio inconveniente, como es el hecho de no estar ponderados; circunstancia que se ha venido excusando por lo que no es sino otra limitación: la toma en consideración de muy pocos productos. La muestra se amplia en los demás índices; sin embargo, éstos se refieren a grupos de bienes determinados por las estadísticas de que proceden (comercio exterior, de cabotaje). Por tanto, aunque ya obedecen a un sistema de ponderación, no pueden aspirar a ser representativos de ningún agregado sectorial.

Cuando el interés del historiador económico se centra en los precios industriales, los problemas se multiplican. El vacío es tal que los índices al por mayor incluyen: tres productos industriales, todos ellos alimentarios, en el caso de Sardá; y seis, en el de la Comisión del patrón oro (CPO), también con predominio de los transformados agrarios. A partir de 1913, con los trabajos de la Dirección General de Estadística, la situación empieza a mejorar sensiblemente ${ }^{4}$; aunque la escasa representación de los precios industriales sólo se corrige de forma satisfactoria para el período posterior a 1955, cuando el Instituto Nacional de Estadística (1960) revisa el sistema de ponderación. Subsisten, pues, las tinieblas aludidas en la cita que abre estas notas. Con todo, merece la pena algún esfuerzo por abrir un pequeño resquicio de luz, siquiera sea tan modesto como éste, hasta que las investigaciones en curso sobre los precios en los siglos XIX y XX nos permitan cubrir una de las lagunas más destacadas en nuestros conocimientos.

La información disponible comprende poco más de dos docenas de series, la mayoría de ellas correspondientes a materias primas industriales y bienes intermedios ${ }^{5}$. En el período 1874-1913, se cuenta con precios para el carbón, mineral de hierro, plomo y plata, piritas, lingote de hierro, acero, metales de plomo, plata, cobre y mercurio, algodón en rama, hilados de algodón, tejidos de algodón, lanas, tejidos de lana, hilaza de lino, manufactura de lino, harina, azúcar, tabaco y aceite. Sin embargo, la heterogeneidad de unidades monetarias y fechas que abarcan las distintas series obliga a una cierta selección.

En ocasiones, como es el caso de los productos minero-metalúrgicos, los datos se refieren a la cotización internacional de productos cuyos precios en el mercado interior también son conocidos. La discrepancia puede atri-

4 Véase, en particular, Dirección General de Estadística (1942). Carreras (1983), pp. 685698, y Maluquer (1989), pp. $521-522$ y 525, ofrecen una panorámica de los índices disponibles.

5 Carreras (1989), pp. 216-235, y Grupo de Estudios de Historia Rural (1989), pp. 115-116. Para la minería y los metales, Coll y Sudrià (1987), pp. 397-483, y Coll (1994). Por más que en la construcción del indice se haya intentado mejorar la cobertura de los productos finales, las fuentes remiten de manera persistente a los precios en origen para grandes transacciones. El que propone este trabajo no puede ser, por tanto, un índice de precios al consumo. 
buirse, en gran parte, a la devaluación de la peseta en el período considerado. Por tanto, el índice que proponemos incluye únicamente los precios interiores ${ }^{6}$. En todo caso, el sesgo introducido está identificado y apunta a una sobrevaloración de la inflación industrial.

Otras veces, los problemas provienen de la falta de continuidad en las series. Así, resulta prácticamente imposible elaborar un índice que recoja cada fase de la producción textil. En este caso, se ha optado por incluir los precios del producto final; lo que contribuye a limitar la dependencia del índice respecto a los bienes intermedios. Para los tejidos de algodón y de lana hemos utilizado información complementaria procedente de Nadal y Sudrià ${ }^{7}$, por un lado, y Esteve Deu ${ }^{8}$, por otro. En las manufacturas de lino, de forma excepcional, ha sido necesario considerar los precios del mercado exterior para todo el período.

La siguiente cuestión consiste en asignar los coeficientes de ponderación adecuados a las distintas series de precios. $\mathrm{El}$ argumento de renunciar a obtener un índice ponderado cuando la muestra es escasa no parece demasiado convincente. Es cierto que la evolución del índice estará condicionada, quizá en exceso, por algunos productos; pero también lo es que, en la economía española del período, los bienes de consumo mantenían un peso considerable en el producto industrial. En otras palabras, la ausencia de ponderación no es neutral. La simple media aritmética exageraría la influencia de la industria básica en el índice. Precisamente la escasez de información, cuando ésta no se distribuye proporcionalmente en los distintos sectores, constituye un argumento a favor de la ponderación, y no en contra.

El cálculo de los coeficientes se lleva a cabo en dos etapas. En primer lugar, a partir del valor añadido generado en 1913, estimamos la importancia relativa entre los distintos productos dentro de su correspondiente sector (minería, metalurgia, textil y alimentación) ${ }^{9}$. Así, la primera columna del

- La desconfianza hacia los precios oficiales declarados en la Estadística Minera aconseja utilizar las rectificaciones de Coll y Sudrià (1987), pp. 420-433, para el carbón, y Coll (1989), pp. 34-52, en otros minerales.

7 Nadal y Sudrià (1993), pp. 206-209, ofrecen los precios de la empresa «La España Industrial, S. A.», para la clase percalina lisa superior, entre 1850 y 1907.

${ }^{8}$ Deu (1986), vol. II, pp. 4-30; citado por Palafox (1991), p. 57.

y En la minería del carbón el valor añadido bruto proviene de Coll y Sudrià (1987), p. 514. Para los productos metalúrgicos ha sido útil la estimación de valores de la producción en Coll (1994), pp. 126-127, y sus coeficientes de transformación: Coll (1985), pp. 131-135. El valor añadido unitario y volumen de producción para las demás series procede generalmente de $\mathrm{Ca}$ rreras (1983), ya que la verosimilitud de sus datos suele verse confirmada al cotejarlos con otras fuentes. No obstante, la producción de aceite de oliva ha sido tomada del Grupo de Estudios 


\section{CUADRO 1}

Valores añadidos relativos y coeficientes de ponderación (\%)

\begin{tabular}{|c|c|c|}
\hline & $I$ & II \\
\hline Minería ........ & 100 . & 11,1 \\
\hline Carbón & 27,7 & 3,1 \\
\hline 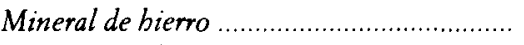 & 39,1 . & 4,3 \\
\hline 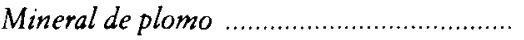 & 21,2 . & 2,4 \\
\hline Piritas de cobre & 12,0 & 1,3 \\
\hline \multicolumn{3}{|l|}{ Metalurgia ............................................ 100} \\
\hline Lingote de bierro ..................................... & 21,9 . & 2,4 \\
\hline 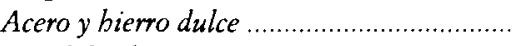 & 39,7 . & 4,4 \\
\hline 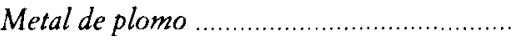 & 38,4 & 4,2 \\
\hline \multicolumn{3}{|c|}{ Textil } \\
\hline Algodón & 70,0 . & 21,3 \\
\hline Lana ....... & 17,9 . & 5,4 \\
\hline 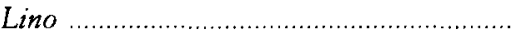 & 12,1 & 3,7 \\
\hline \multicolumn{3}{|l|}{ Alimentación ............................................ 100} \\
\hline Harina & 31,8 . & 15,1 \\
\hline Tabaco. & 34,4 . & 16,3 \\
\hline Aceite.. & 20,0 . & 9,5 \\
\hline Azúcar. & 13,8 . & 6,6 \\
\hline
\end{tabular}

FUENTE: La columna $I$ se ha elaborado con los datos a que se refiere la nota al pie 10. En la columna $I I$, los porcentajes sectoriales proceden de Prados (1988), p. 163, aunque han sido corregidos proporcionalmente para cubrir la ausencia de datos sobre precios en las industrias química, papelera y de la piel. Los coeficientes de ponderación para cada producto se obtienen como explica el texto.

cuadro 1 muestra la relación que deben mantener entre sí las ponderaciones del algodón, la lana y el lino, por ejemplo, para que reflejen su aportación relativa al valor añadido industrial en el año base. Pero nada nos dice acerca de la proporción que éstas han de guardar, digamos, con los productos

de Historia Rural (1991), p. 1190; y para el mineral de hierro parecen preferibles los beneficios y costes salariales unitarios de Escudero (1994), pág. 87. En el caso de la industria algodonera, se ha estimado el valor añadido unitario a partir del que se propone en Nadal y Sudrià (1993); si bien considerando, de acuerdo con Maluquer (1994), pp. 51-52, que la merma del 10 por ciento en el proceso de hilatura y tisaje se habría reducido aproximadamente a la mitad como consecuencia del cambio técnico. Sobre el consumo aparente de hilados se ha practicado la misma corrección. El peso relativo entre el algodón y la lana resulta, entonces, más coherente con el que obtiene el propio Maluquer, en su índice de la producción industrial catalana, y el que se deduce de Parejo (1992), para el conjunto español. 


\section{CUADRO 2}

Indice ponderado de precios industriales $(1913=100)$

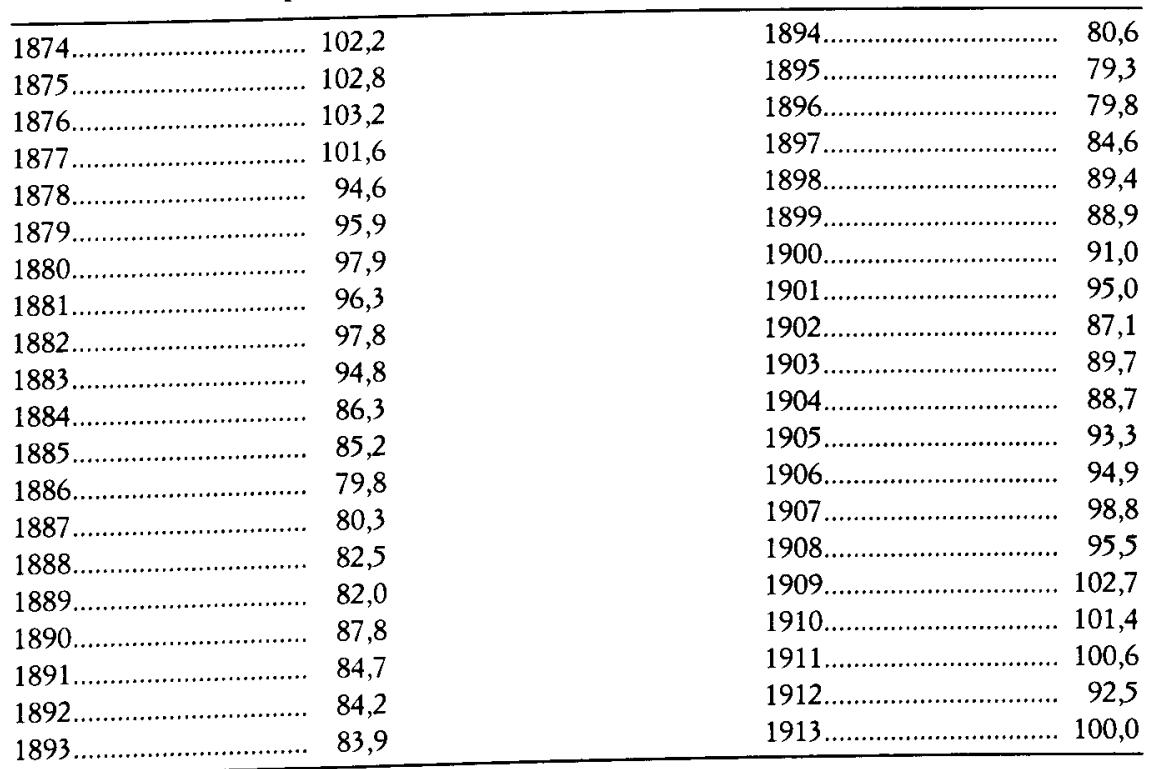

FUENTE: Elaborado según explica el texto.

metalúrgicos. Para ello se requiere, en segundo lugar, alguna indicación sobre el peso de cada rama en el conjunto de la industria. Leandro Prados recoge esta información para 1900 a partir de las contribuciones industriales analizadas por $\mathrm{Nadal}^{10}$. Al multiplicar estos porcentajes (subtotales de la col. II) por los de participación relativa en el valor añadido sectorial, obtenemos los coeficientes para las diferentes series ${ }^{11}$ cuya media ponderada constituye nuestra aproximación al comportamiento de los precios industriales en la España de entresiglos (Cuadro 2).

10 Prados (1988), pp. 143-168; y Nadal (1987), pp. 51-58.

11 Nadal y Sudrià (1993): tejidos de algodón, 1, pp. 208-209. Palafox (1991): tejidos de lana, 2 (1900-1913), p. 57. Coll y Sudrià (1987): carbón, p. 432. Grupo de Estudios de Historia Rural (1989): aceite, 479, pp. 115-116. Coll (1989): mineral de hierro, pp. 44-45; mineral de plomo, pp. 47-48; pirita de cobre, p. 51. Carreras (1989): lingote de hierro, 882, pp. 225-226; acero y hierro dulce, 884 , pp. 225-226; metal de plomo, 878 , pp. 222-223; tejidos de lana, 894 (1874-1900), p. 229; manufacturas de lino, 897, pp. 229-230; harina de trigo, 898, pp. 232-233; tabaco, 901, pp. 232-233; azúcar refinado, 899, pp. 232-233. 
La presentación del nuevo indice ponderado de precios industriales (IPPI) exige al menos un somero cotejo con el Índice de Precios al por Mayor (IPM) de Sardá y la Comisión del patrón oro ${ }^{12}$. La comparación se ha llevado a cabo previa transformación logarítmica de ambos. Hemos efectuado ajustes mínimo-cuadráticos por períodos seleccionados con el fin de captar la tendencia (gráficos 1 a 4):

$$
\log I=a+b T
$$

donde $I$ es el índice correspondiente y $T=1,2 \ldots$, el tiempo.

$\mathrm{El}$ antilogaritmo de la pendiente estimada constituye un valor alternativo para la tasa de crecimiento anual de los precios, con la ventaja de recoger información procedente de todos los años. En la medida que el IPM incluye fundamentalmente precios agrarios ${ }^{13}$, la diferencia entre las columnas 1 y 2 del cuadro 3 puede interpretarse como una aproximación a la relación real de intercambio entre la agricultura y la industria.

\section{CUADRO 3}

Tasas de inflación interanual, 1874-1913 (\%)

\begin{tabular}{crrc}
\hline & $(1)$ & $(2)$ & $(3)$ \\
& $I P M$ & $I P P I$ & $(1)-(2)$ \\
\hline $1874-1890$ & $-1,1$ & $-1,6$ & 0,5 \\
$1890-1913$ & 1,4 & 0,9 & 0,5 \\
\hline
\end{tabular}

FUENTE: Véase el texto.

Los primeros años de la década final del siglo pasado, con la aplicación de medidas comerciales restrictivas, marcan una inflexión al alza de los precios tanto en un índice como en otro; pero el IPM arroja una inflación anual, entre 1890 y 1913, claramente por encima del IPPI. Del mismo modo, antes de 1891, cuando la tendencia es a la baja, los precios industriales descienden a un ritmo que supera en medio punto la media del

12 Maluquer (1989), p. 518.

${ }_{13}$ Hasta 1890, nueve productos: arroz, aceite de oliva, cebada, harina, trigo, azúcar, algodón, café y cacao. Desde entonces a 1913, hay que añadir: alcohol, almendra, bacalao, carbón, cobre, habichuelas, maíz y petróleo. 


\section{GRÁFICO 1}

IPPI, 1874-1890

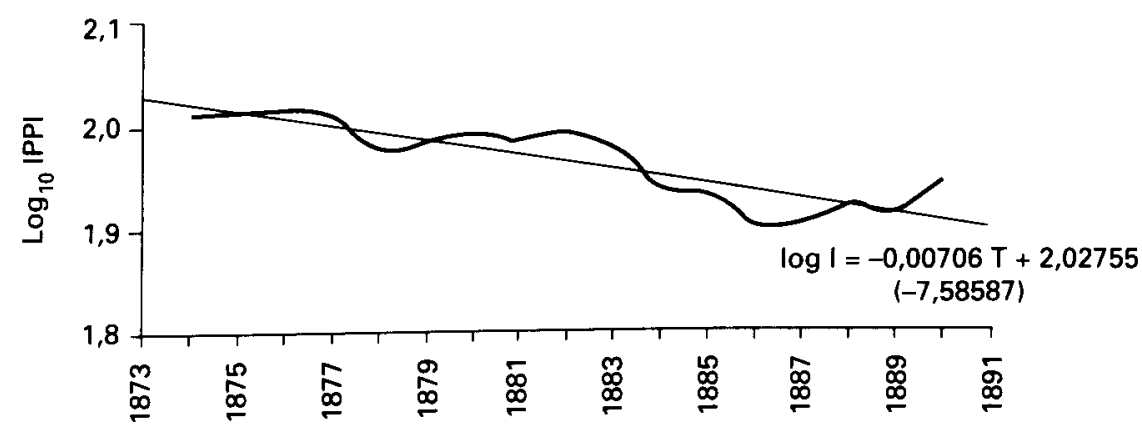

GRÁFICO 2

IPM, 1874-1890

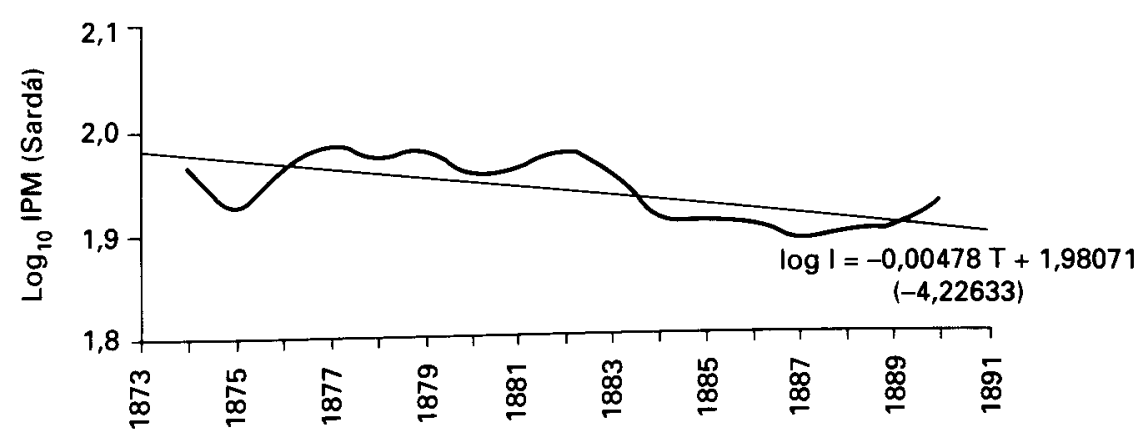

IPM ${ }^{14}$. Asi pues, los términos de intercambio evolucionaron siempre a favor del sector agrario.

La repercusión negativa de unos alimentos caros, acordes con una agricultura de baja productividad, sobre la demanda de productos manufacturados, y en general, sobre el crecimiento y la modernización económica

14 Los valores correspondientes a la tercera columna del cuadro 3 se obtienen, en realidad, a partir de la pendiente estimada en el ajuste de $\log$ (IPM / IPPI) a una tendencia lineal: (anti$\log b-1) \times 100 ;$ donde $b_{1874-1490}=0,00228$ y $b_{1891 \cdot 1913}=0,00196$, cuyos estadísticos $t$ son 2,54488 y 2,53998 , respectivamente. 


\section{GRÁFICO 3}

IPPI, 1890-1913

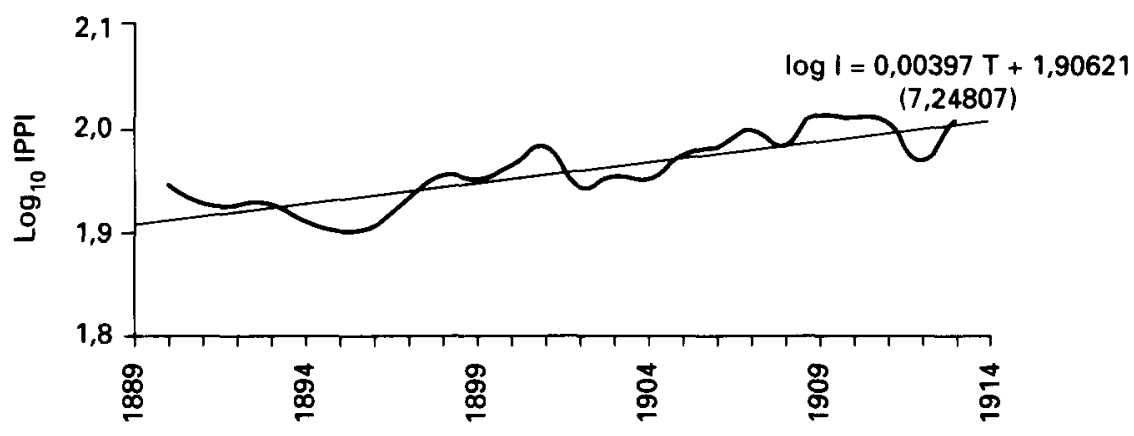

GRÁFICO 4

IPM, 1890-1913

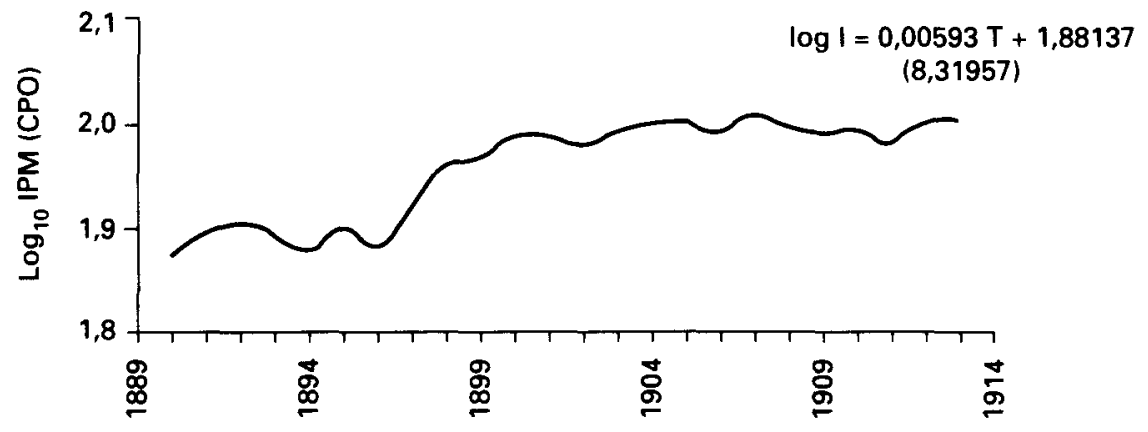

del país, fue bien establecida por Jordi Palafox ${ }^{15}$. Con todo, el énfasis recaía en la trayectoria alcista de los precios agrarios españoles, por encima de la tendencia en el mercado internacional. Desde estas páginas se ha pretendido aportar material para un tratamiento más explícito de otra variable relevante, como los precios de nuestro propio sector industrial. De hecho, su comportamiento tiende a confirmar la rémora que supuso una agricultura atrasada y protegida en el proceso de industrialización.

15 Véase, Palafox (1991); especialmente, el capítulo 1 y la nota II de los apéndices. 


\section{BIBLIOGRAFÍA}

ARENALES, M. ${ }^{a}$ C. (1976): «Un indicador de precios de la economía española para el período 1850-1900», en Datos básicos para la Historia Financiera de España, 1850-1975, vol. I, Madrid, Instituto de Estudios Fiscales, pp. 1109-1123.

Bustelo, F., y TORTELLA, G. (1976): «Monetary Inflation in Spain, 1800-1970», Journal of European Economic History, vol. 5, n. ${ }^{\circ} 1$, pp. 141-150.

CARRERAS, A. (1983): La producció industrial espanyola $i$ italiana des de mitjan segle XIX fins a l'actualitat, Tesis Doctoral, Universidad Autónoma de Barcelona. (1989): «La industria», en A. Carreras (coord.): Estadísticas históricas de España. Siglos XIX-XX, Madrid, Fundación Banco Exterior, pp. 169-247.

COLL, S. (1985): «Proyecto "Producción, productividad y renta de las economías de Europa Occidental, 1789-1956". Informe sobre producción y valor añadido de los sectores minero y metalúrgico», mecanografiado.

- (1989): «¿Explotación minera en o de España? Una especulación», ponencia presentada al IV Congreso de la Asociación de Historia Económica, Alicante. (1994): «Precios y valores de la producción minera y metalúrgica española, 1968-1935. Un intento de rectificación de las cifras oficiales», Áreas, n. 16, pp. 95-128.

COLL, S., y SUDRIÀ, C. (1987): El carbón en España, 1770-1960. Una bistoria económica, Madrid, Turner.

Deu i BAIGUAL, E. (1986): La indústria llanera a Sabadell en el primer quart del segle XX, Tesis Doctoral, Universidad Autónoma de Barcelona.

Dictamen de la Comisión nombrada por Real Orden de 9 de enero de 1929 para el es tudio de la implantación del patrón oro, Madrid, Consejo Superior Bancario, 1929; reproducido en Información Comercial Española, 318, febrero de 1960, pp. 51-83.

DIRECCIÓN GENERAL DE ESTADÍSTICA (1942): «Precios al por mayor y números índice, 1913-1941», Boletín de Estadística, número extraordinario.

ESCUDERO, A. (1994): «Valores añadidos de la minería vizcaína, 1876-1936», Áreas, n. ${ }^{\circ} 16$, pp. $79-91$.

GRUPO DE ESTUDIOS DE HISTORIA RURAL (1989): «El sector agrario hasta 1935», en A. Carreras (coord.): Estadísticas históricas de España. Siglos XIX-XX, Madrid, Fundación Banco Exterior, pp. 91-129.

(1991): Estadisticas bistóricas de la producción agraria española, 1859-1935, Madrid, Ministerio de Agricultura, Pesca y Alimentación.

INSTITUTO NACIONAL DE ESTADÍSTICA (1960): Precios al por mayor. Nuevo sistema de números índices. Año base: 1955, Madrid.

MALUQUER DE MOTES, J. (1989): «Precios, salarios y beneficios. La distribución funcional de la renta», en A. Carreras (coord.): Estadisticas históricas de España. Siglos XIX-XX, Madrid, Fundación Banco Exterior, pp. 495-532. (1994), «El índice de la producción industrial de Cataluña. Una nueva estimación (1817-1935)», Revista de Historia Industrial, n. ${ }^{\circ}$ 5, pp. 45-71.

NADAL, J. (1987): «La industria fabril española en 1900. Una aproximación», en NADAL; J., CARRERAS, A., y SUDRIÀ, C. (comp.): La economía española en el siglo XX Una perspectiva bistórica, Barcelona, Ariel, pp. 23-61. 
NADAL, J., y SudRIÀ, C. (1993): «La controversia en torno al atraso económico español en la segunda mitad del siglo XIX (1860-1913)», Revista de Historia Industrial, n. 3 , pp. 199-227.

PAlafox, J. (1991): Atraso económico y democracia. La Segunda República y la economía española, 1892-1936, Barcelona, Crítica.

PAREJO, A. (1992): «La industria lanera en la Europa del sur: un análisis comparativo», Revista de Historia Industrial, n. ${ }^{\circ} 2$, pp. 87-119.

PRADOS DE LA EscosURA, L. (1982): Comercio exterior y crecimiento económico en España, 1826-1913. Tendencias a largo plazo, Madrid, Banco de España.

(1988): De imperio a nación. Crecimiento y atraso económico en España (17801930), Madrid, Alianza.

SARDÁ, J. (1948): La política monetaria y las fluctuaciones de la economia española en el siglo XIX, Madrid, Instituto Sancho de Moncada de Economía del CSIC; reeditado por Ariel, Barcelona, 1970. 